\title{
Charge Manipulation in Molecules Encapsulated Inside Single-Wall Carbon Nanotubes
}

\author{
Kazuhiro Yanagi* and Rieko Moriya \\ Department of Physics, Tokyo Metropolitan University, Tokyo 192-0397, Japan \\ Nguyen Thanh Cuong and Minoru Otani \\ Nanosystem Research Institute (NRI), National Institute of Advanced Industrial Science and Technology (AIST), \\ Tsukuba 305-8568, Japan \\ Susumu Okada \\ Graduate School of Pure and Applied Sciences, University of Tsukuba, 1-1-1 Tennodai, Tsukuba 305-8571, Japan
}

(Received 11 July 2012; published 19 February 2013)

\begin{abstract}
We report clear experimental evidence for the charge manipulation of molecules encapsulated inside single-wall carbon nanotubes (SWCNTs) using electrochemical doping techniques. We encapsulated $\beta$-carotene (Car) inside SWCNTs and clarified electrochemical doping characteristics of their Raman spectra. $\mathrm{C}=\mathrm{C}$ streching modes of encapsulated Car and a $G$ band of SWCNTs showed clearly different doping behaviors as the electrochemical potentials were shifted. Electron extraction from encapsulated Car was clearly achieved. However, electrochemical characteristics of Car inside SWCNTs and doping mechanisms elucidated by calculations based on density-functional theory indicate the difficulty of charge manipulation of molecules inside SWCNTs due to the presence of strong on-site Coulomb repulsion energy at the molecules.
\end{abstract}

DOI: 10.1103/PhysRevLett.110.086801

PACS numbers: 73.22. $-\mathrm{f}$

According to Gauss's law of electromagnetism, surface charges on conductive cylindrical tubes cannot induce electric fields inside hollow spaces. Therefore, charges on the materials located inside the cavity and electrically separated from the surface cannot be influenced by the amount of surface changes present [1]. In general, surface charges are distributed to a depth of approximately $1 \mathrm{~nm}$ from the surface [2]. In this context, we are interested in determining what happens when the size of the tubes becomes "nano," such that the diameter of the cylinder is approximately $1 \mathrm{~nm}$. Single-wall carbon nanotubes (SWCNTs) are cylindrical graphitic tubes, with a diameter of approximately $1.0 \mathrm{~nm}$, that can encapsulate various types of molecules. The electrochemical doping technique can introduce negative or positive surface charges on the nanotubes through the formation of electric double layers on their surfaces. Several studies have performed electrochemical doping upon SWCNTs encapsulating $\pi$-conjugated molecules; however, it is still unclear whether the charges of the molecules encapsulated inside the nanocavity can be controlled by the formation of the electric double layers on the nanotube surfaces [3-6]. One study found it difficult to manipulate the charges of the encapsulated molecules [5], but the other study implied some influence of electrode potential upon molecules, although complete charge transfer was ruled out [3]. Herein, we report clear experimental evidence for the charge manipulation of encapsulated molecules using electrochemical doping techniques. In addition, we theoretically elucidated the associated mechanism. We clarified that the encapsulated $\pi$-conjugated molecules, such as $\beta$-carotene (Car), can be electrochemically hole doped (i.e., electron extracted) using electrochemical doping processes. When one electron was extracted from Car, the highest occupied molecular orbital (HOMO) of Car substantially shifted downward and crossed the highest branch of the valence band of the semiconducting SWCNT. In this situation, the electrons were primarily extracted from the SWCNTs. The large on-site Coulomb energy of Car leads to a substantial downward shift of the HOMO of Car, thereby preventing electron extraction from the state.

In this study, Car was employed as the encapsulated molecule for the following three reasons. (1) Car has a simple linear polyene structure, which is a model for onedimensional $\pi$-conjugated molecules [a schematic structure is shown in Fig. 1(a)]. (2) The process of encapsulating Car into SWCNTs is well established [7-9]. The presence of Car inside SWCNTs has been confirmed by x-ray and Raman [8,9] and by transmission electron microscope measurements (the presence of retinal, whose structure is half of Car, was clearly identified) [7]. (3) The Raman signal of Car, such as the $\mathrm{C}=\mathrm{C}$ stretching mode (the $\nu_{1}$ mode), inside SWCNTs is relatively strong and comparable to the intensity of the $G$ band of the surrounding semiconducting SWCNTs (Semi) [9]. This unique situation results from the electronic band configuration. The optical absorption of encapsulated Car is located in the $S_{33}$ and $S_{44}$ optical absorption region ( $i$ indicates the $i$ th optical transition) of the Semi [9]. Therefore, the excitation wavelength (i.e., $488 \mathrm{~nm}$ ) in this region is simultaneously in 
resonance with Semi and Car, leading to the strong Raman signals from both Semi and Car. These strong Raman signals enable us to simultaneously evaluate doping properties of both Semi and encapsulated Car due to the strong relationship between the Raman spectra and the doping states of the SWCNTs and Car.

The Raman spectra of SWCNTs are very sensitive to electron or hole injection [10]. The influence of electrochemical doping on the Raman spectra of the $G$ band of semiconducting SWCNTs has been thoroughly studied [10]. Hole and electron injections to Semi induce a decrease in the intensity and a shift in the $G$ band, as shown in Fig. S1 of the Supplemental Material [11]. In the case of Car, Car can be oxidized when the potential is positive, and the dications and cation radicals of Car are formed by the oxidation $[12,13]$. The optical absorption of neutral Car disappeared during the oxidation (Fig. S2 of the Supplemental Material [11]). Therefore, the intensity of the $\nu_{1}$ mode of Car decreased due to the oxidation (Fig. S3 of the Supplemental Material [11]) because the disappearance of the optical absorption of neutral Car resulted in an off-resonance state of the Raman excitation wavelength. Therefore, it is possible to evaluate the doping process from the changes in the Raman spectra. In addition, encapsulated Car is stable during Raman measurements (i.e., no polymerization occurs, unlike the case of encapsulated fullerenes, the polymerization reactions of which can decrease the Raman signals [14]).

In this study, to clarify the doping mechanism of Car surrounded by a specified electronic structure of SWCNTs, Car encapsulated in Semi (Car@Semi) was prepared by density-gradient purification methods $[15,16]$. For sample preparation, SWCNTs with an average diameter of $1.4 \mathrm{~nm}$, which were produced by arc-discharge methods (ArcSO, Meijyo), were used as starting materials. The optical absorption spectrum of Car@Semi is shown in Fig. S4 of the Supplemental Material [11]. The Semi was chosen due to its simultaneous resonance with Car, as mentioned above. Electrochemical doping measurements were performed on a film of Car@Semi. Typical electrochemical doping techniques were employed to introduce charges on the SWCNTs encapsulating Car [17]. Figure 1 shows a schematic representation of the experimental setup for the electrochemical doping. $\mathrm{An} \mathrm{Ag} / \mathrm{Ag}^{+}$reference electrode was employed for the reference electrode. Pt was employed for the contact and counterelectrodes. To apply a wider potential, an ionic liquid $[N, N$, $\mathrm{N}$-trimethyl- $\mathrm{N}$-propylammonium bis(trifluoromethanesulfonyl)imide] was used for measurements of the SWCNT films. Tetrabutylammonium hexafluorophosphate and anhydrous dichloromethane were employed for Car in solution. The oxidation potential of ferrocene was employed for normalization purposes to evaluate potential dependencies in the ionic liquid and the dichloromethane solutions. The SWCNT films were formed on a glass
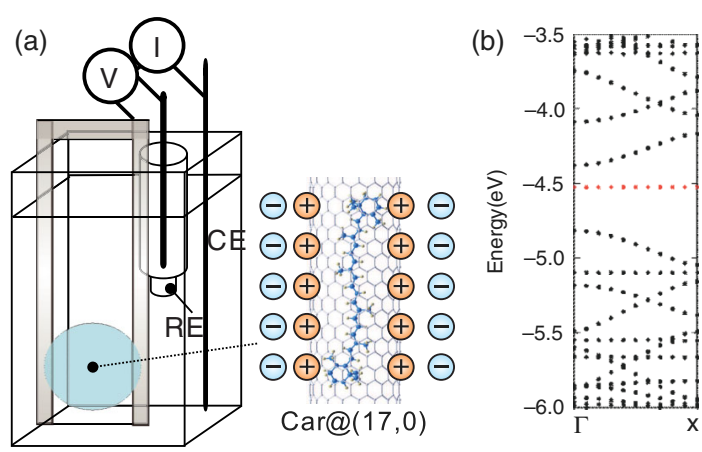

FIG. 1 (color online). (a) A schematic representation of the experimental setup for the electrochemical doping of Car@(17,0). RE and CE are reference and counter electrodes, respectively. (b) The band structure of Car@(17,0) calculated using DFT. Red dots represent the HOMO band associated with Car, and the black dots correspond to $(17,0)$.

substrate and attached to the Pt electrodes. The potential of the films was controlled using a potentiostat. When the potential was shifted to negative or positive, electric double layers were formed on the surface of the SWCNTs, thus introducing negative (electrons) or positive (holes) charges to the SWCNTs. Raman spectra were measured using a single monochromator system (Acton, 2300i) equipped with a charge-coupled-device detector (PIXIS, Princeton), an edge filter (Semrock), and an $\mathrm{Ar}^{+}$laser (Spectra Physics, 2017) operated at $488 \mathrm{~nm}(2.54 \mathrm{eV})$. All measurements were performed at room temperature. Figure 1(b) shows the electronic structure of Car@ $(17,0)$ SWCNTs obtained from first-principles calculations based on density-functional theory (DFT). The details of the calculation can be found in the Supplemental Material [11]. In the figure, the HOMO of Car is located in the gap of $(17,0)$ SWCNTs. Therefore, it is expected that, when the potential is shifted in the positive direction, the electrons of Car will be extracted, followed by the electrons of $(17,0)$. However, the experimental results were not as simple and are described below.

Figure 2 shows the changes in the Raman spectra of Car@Semi as a function of the potential voltages. At zero potential (versus $\mathrm{Ag} / \mathrm{Ag}^{+}$), we can clearly identify the Raman peaks due to the $\mathrm{C}=\mathrm{C}$ stretching mode of Car ( $\nu_{1}$ mode) and the $G$ band $\left(G^{+}\right.$and $G^{-}$) of Semi. When the potential was shifted in the negative direction (i.e., electron injection), the intensity of the $G$ band decreased and no significant change was observed in the $\nu_{1}$ mode [Fig. 2(a)]. These results indicate that electron injection occurred in the SWCNTs but not in Car. According to the electronic band in Fig. 1(b), the electrons were introduced to the conduction band of the Semi. The calculation suggests that the lowest unoccupied state of Car is located significantly above the conduction band of the Semi (not seen in the figure). Therefore, no electrons were introduced to the encapsulated Car.

However, when the potential was shifted in the positive direction (i.e., electron extraction), the intensities of both 

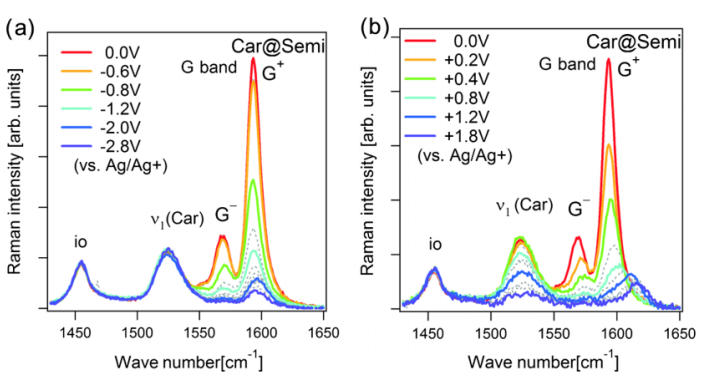

FIG. 2 (color online). Raman spectra of Car@Semi as a function of the potential voltages [(a) negative, (b) positive]. The spectra were obtained at a $488 \mathrm{~nm}$ excitation wavelength. "io" indicates the Raman peak of ionic liquid used in the measurements. The dotted lines are Raman spectra at potentials between those described with the colored solid lines.

the $G$ band and the $\nu_{1}$ mode varied [Fig. 2(b)]. When the potential was shifted to $+0.4 \mathrm{~V}$, the intensity of the $\nu_{1}$ mode was nearly the same as the initial intensity. However, the intensity of the $G$ band had decreased to half of the intensity at $0 \mathrm{~V}$. At $+0.8 \mathrm{~V}$ in Fig. 2(b), the intensity of the $\nu_{1}$ mode began to decrease. Then, at $1.6 \mathrm{~V}$, both the $\nu_{1}$ mode and the $G$ band nearly disappeared. The decreased Raman signals of both the $G$ band and the $\nu_{1}$ band were recovered when the potential was returned (see Fig. S5 of the Supplemental Material [11]).

The intensities of the $G^{+}$band and the $\nu_{1}$ mode as a function of the potential voltages are shown in Fig. 3(a). The potential dependence of the intensities of the $\nu_{1}$ modes of Car in Semi is significantly different from that of Car in solution [Fig. 3(c)]. At $0.4 \mathrm{~V}$ (versus Fc $/ \mathrm{Fc}^{+}$) in Fig. 3(c), the intensity of the $\nu_{1}$ mode of Car in solution nearly disappeared. However, the intensity of the mode in Semi only decreased slightly. It is important to note that the decline in the mode of Car in Semi at a positive potential was gradual compared with the steep decline in the mode of Car in solution. This result suggests that the surrounding nanotube walls significantly influenced the charge extraction processes of the encapsulated molecules. But, there was no significant difference between the potential dependence of the $G^{+}$band in Semi with and without Car [Fig. 3(b)], suggesting that the presence of Car did not so much influence the charge extraction processes of the surrounding SWCNTs.

The dependence of the $G$ band and the $\nu_{1}$ mode on the potential [Fig. 3(a)] exhibited an unexpected behavior with regard to the electronic band structure of Car@ $(17,0)$. The $G$-band signal decreased first, followed by the $\nu_{1}$ mode signal, suggesting that electron extraction occurred from Semi and then from Car, which is in contrast to what was expected based on the band structure in Fig. 1(b). To understand the details of the electron extraction mechanism for encapsulated Car, additional DFT calculations were performed on Car@(17,0), which was subjected to an electric field (Fig. 4). All of the calculations employed DFT using the effective screening medium methods to simulate the electric field applied to the Car@(17,0). To simulate the Car@(17,0) under the electric double layer condition, we considered the structural model in which the Car@(17,0) is placed upon a two-dimensional electrode separated by $1 \mathrm{~nm}$ from the wall of a $(17,0)$ SWCNT [Fig. 4(a)]. The calculations showed that the HOMO shifts downward due to an increase in the electric field or the number of holes (i.e., the number of extracted electrons). Then, the HOMO crosses the top of the valence band of the Semi under the electric field [Fig. 4(b)]. From the results of the calculations, the following electron extraction mechanisms are deduced. The Fermi energy of Car@Semi was downshifted by the positive shift of the potential voltage. The electrons of the valence electronic bands of Semi located above the Fermi energy were totally extracted. However, the electrons of Car were just partially extracted from HOMO due to the downward shift of HOMO, which followed the shift of the Fermi energy and was located at the Fermi energy position. Thus, electrons would first be extracted mainly from Semi and then from Car.

The substantial downward shift in the HOMO of Car was attributed to the large on-site Coulomb repulsion energy arising from the size and dimensionality of the Car. The on-site energy $U$ is evaluated by using the formula
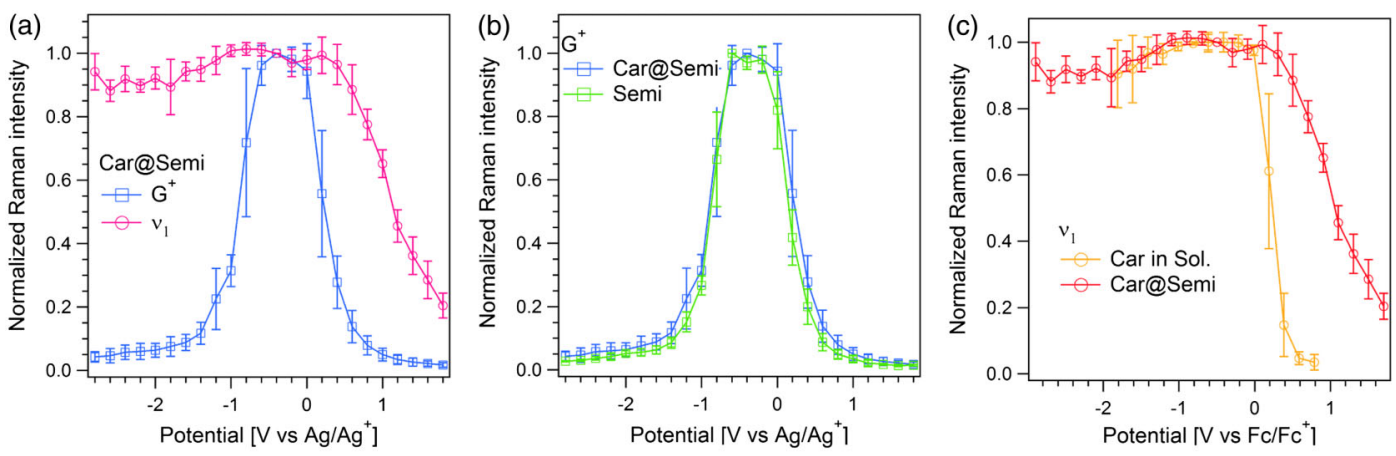

FIG. 3 (color online). Comparison of the potential dependence of the Raman intensities (a) between the $G^{+}$band (blue curve) and the $\nu_{1}$ mode (red curve) in Car@Semi, (b) between the $G^{+}$bands of Car@ Semi (blue curve) and Semi (green curve), and (c) between the $\nu_{1}$ modes of Car in solution (Sol.) (yellow curve) and Car@Semi (red curve). 

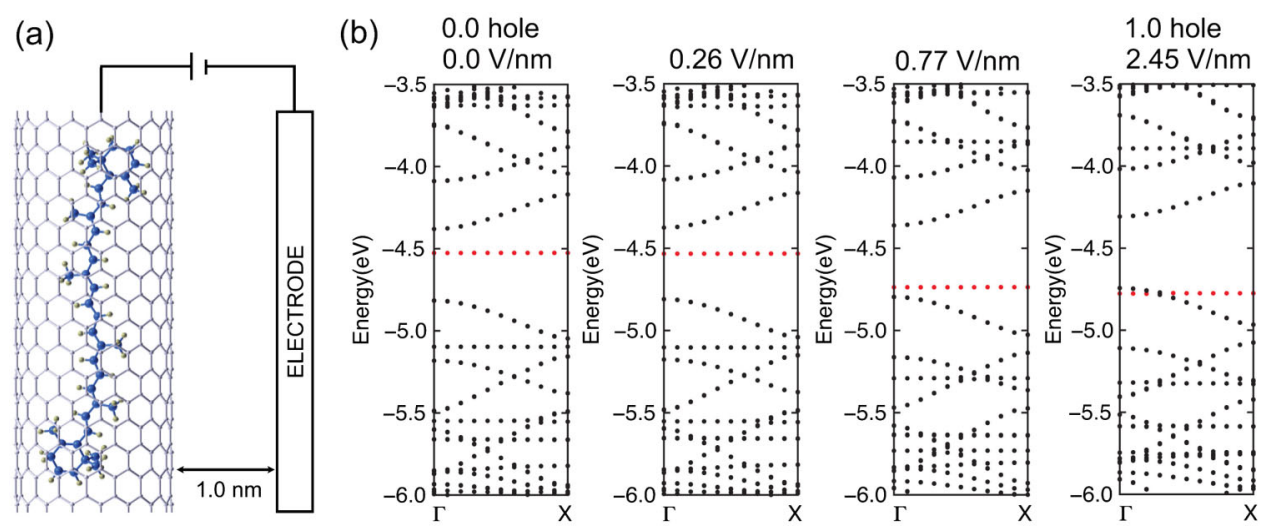

FIG. 4 (color online). (a) Schematic representation of the calculations. (b) Electronic structures of Car@ $(17,0)$ under various electric fields. The red dots correspond to the HOMO band of Car, and the black dots correspond to $(17,0)$. At $2.45 \mathrm{~V} / \mathrm{nm}$, one electron was extracted from Car.

proposed by Anisimov et al. [18]: $U=[E(N)-E(N-1)]-$ $[E(N-1)-E(N-2)]$, where $E(N+m)$ is the total energy for $N+m$ electrons evaluated by DFT calculations. The $1 \mathrm{~nm}$ size of the molecule leads to a large on-site energy for the occupied states. The calculated on-site Coulomb energy was a few hundred $\mathrm{meV}$, which is substantial because the value is close to that of the dangling bond on $\mathrm{Si}(111)$ surfaces $(U=0.7 \mathrm{eV})[19]$. Therefore, the electron extraction from the states results in a substantial downward shift with a decrease in energy. In sharp contrast, the $\pi$ electron states of the SWCNT extended throughout the nanotube axis of micrometer length. Therefore, the eigenvalue of the electronic states of the SWCNTs is insensitive to the electron extractions or injections and maintains its neutral energy.

The presence of such strong on-site Coulomb repulsion qualitatively explains the physical background of the observed phenomena. The electronic band structure of Semi did not change if the Fermi energy was downshifted, but the HOMO band of Car would go down following the shift of the Fermi energy due to the strong on-site Coulomb repulsion. As shown in Fig. 3(c), a gradual decrease of the intensity of the $\nu_{1}$ mode of Car@Semi is a remarkable contrast to a sharp decrease of that of Car in solution at positive potential. The decrease of the intensity of the $\nu_{1}$ mode is related to the amount of extracted charges of Car. Thus, the experimental results suggest that the removal of electrons from Car was rather gradual in Car@Semi. We assume that one of the origins for such gradual extraction would be due to the continuous downward shift of the HOMO.

The results also suggest why the reduction of $\mathrm{C}_{60}$ encapsulated in SWCNTs was reported to be difficult $[5,6]$. The on-site Coulomb energy of $\mathrm{C}_{60}$ is several times larger than that of Car, resulting in the lowest unoccupied molecular orbital of $\mathrm{C}_{60}$ being higher than the conduction band of the SWCNT under electron injection. Therefore, electron doping of $\mathrm{C}_{60}$ is difficult due to the continuous introduction of electrons into the SWCNT.
In summary, we experimentally elucidated the processes of electron extraction from Car encapsulated inside SWCNTs using electrochemical doping techniques. The presence of the surrounding SWCNTs significantly influenced the processes of electron extraction from encapsulated Car. Theoretical calculations suggested that the HOMO band of Car was located below the valence band of the SWCNTs when the Fermi energy was shifted. The calculations revealed the presence of a strong on-site Coulomb repulsion energy on Car. Therefore, the electron extraction from the encapsulated Car was relatively more difficult than that from the SWCNTs.

This work was partially supported by a Grant-in-Aid for Scientific Research on Innovative Areas (No. 21108523, " $\pi$-Space") and Grant No. 2365117 from the Ministry of Education, Culture, Sports, Science, and Technology; by the Industrial Technology Research Grant Program in 2007 from the New Energy and Industrial Technology Development Organization; and by CREST, Japan.

*yanagi-kazuhiro@tmu.ac.jp

[1] R. Feynman, R. Leighton, and M. Sands, The Feynman Lectures on Physics (Addison-Wesley, Reading, MA, 1977).

[2] A. Pandolfo and A. Hollenkamp, J. Power Sources 157, 11 (2006).

[3] M. Kalbac, L. Kavan, S. Gorantla, T. Gemming, and L. Dunsch, Chem. Eur. J. 16, 11753 (2010).

[4] M. Kalbac, L. Kavan, M. Zukalova, and L. Dunsch, J. Phys. Chem. C 111, 1079 (2007).

[5] L. Kavan, L. Dunsch, H. Kataura, A. Oshiyama, M. Otani, and S. Okada, J. Phys. Chem. B 107, 7666 (2003).

[6] L. Kavan, L. Dunsch, and H. Kataura, Chem. Phys. Lett. 361, 79 (2002).

[7] Z. Liu, K. Yanagi, K. Suenaga, H. Kataura, and S. Iijima, Nat. Nanotechnol. 2, 422 (2007).

[8] K. Yanagi, K. Iakoubovskii, S. Kazaoui, N. Minami, Y. Maniwa, Y. Miyata, and H. Kataura, Phys. Rev. B 74, 155420 (2006). 
[9] K. Yanagi, Y. Miyata, and H. Kataura, Adv. Mater. 18, 437 (2006).

[10] L. Kavan, P. Rapta, L. Dunsch, M. J. Bronikowski, P. Willis, and R. E. Smalley, J. Phys. Chem. B 105, 10764 (2001).

[11] See Supplemental Material at http://link.aps.org/ supplemental/10.1103/PhysRevLett.110.086801 for the experimental results of electrochemical doping upon films of semiconducting SWCNTs and Car in a dichloromethane solution, optical absorption spectrum of Car@Semi, recovery of Raman-intensities of G-band of Car@Semi when the potentials were shifted, the details of the density functional theory calculations, and the atomic coordinate of the Car encapsulated in $(17,0)$ SWCNT.

[12] J. Jeevarajan, C. C. Wei, A.S. Jeevarajan, and L.D. Kispert, J. Phys. Chem. 100, 5637 (1996).

[13] D. Liu, Y. Gao, and L. Kispert, J. Electroanal. Chem. 488, 140 (2000).
[14] H. Kataura, Y. Maniwa, M. Abe, A. Fujiwara, T. Kodama, K. Kikuchi, H. Imahori, Y. Misaki, S. Suzuki, and Y. Achiba, Appl. Phys. A 74, 349 (2002).

[15] K. Yanagi, Y. Miyata, Z. Liu, K. Suenaga, S. Okada, and H. Kataura, J. Phys. Chem. C 114, 2524 (2010).

[16] K. Yanagi, H. Udoguchi, S. Sagitani, Y. Oshima, T. Takenobu, H. Kataura, T. Ishida, K. Matsuda, and Y. Maniwa, ACS Nano 4, 4027 (2010).

[17] K. Yanagi, R. Moriya, Y. Yomogida, T. Takenobu, Y. Naitoh, T. Ishida, H. Kataura, K. Matsuda, and Y. Maniwa, Adv. Mater. 23, 2811 (2011).

[18] V. I. Anisimov, I. V. Solovyev, M. A. Korotin, M. T. Czyzyk, and G. A. Sawatzky, Phys. Rev. B 48, 16929 (1993).

[19] S. Okada, K. Shiraishi, and A. Oshiyama, Phys. Rev. Lett. 90, 026803 (2003). 